\title{
UNA ALTERNATIVA AL MODELO SANITARIO TRADICIONAL: CASO MUFACE
}

\author{
Autor: José Manuel López Saludas ${ }^{1}$ \\ Profesor Asociado \\ Departamento de Economía y Empresa \\ Facultad de Ciencias Económicas y Empresariales \\ Universidad Pompeu Fabra
}

\section{Resumen}

El modelo sanitario MUFACE posibilita la libertad de elección. Este modelo sanitario no prejuzga si la sanidad pública es mejor que la privada o viceversa, ya que MUFACE ofrece a sus mutualistas tanto servicios sanitarios producidos por empresas públicas como por empresas privadas. La cobertura garantizada por las empresas aseguradoras privadas son equivalentes a las de la sanidad pública (la Cartera Común de Servicios del SNS) e incluso más prestaciones. Dicho modelo es más eficiente (la sanidad pública produce los mismos servicios sanitarios entre un $40 \%$ y $60 \%$ más caro que el sector privado), proporciona al usuario mayor libertad (libertad de elección de médico, especialista y centro hospitalario) y mayor accesibilidad (no existen listas de espera).

\footnotetext{
${ }^{1}$ lopezsaludas@hotmail.com
} 
Palabras clave: educación; sanidad; eficacia; eficiencia; ineficiencia; productividad; sistema sanitario

\title{
An alternative to traditional health care model: case MUFACE
}

\begin{abstract}
MUFACE health model enables freedom of choice. The medical model does not prejudge whether public health is better than private or vice versa, since mutual MUFACE offers both health services produced by public companies and private companies. Guaranteed coverage by private insurance companies are equivalent to those of public health (the Common Services Portfolio SNS) and even more features. This model is more efficient (public health produces the same health services between $40 \%$ and $60 \%$ more expensive than the private sector), it gives users greater freedom (freedom of choice of doctor, specialist or hospital) and increased accessibility (there are no waiting lists).
\end{abstract}

Key words: merit goods; effectiveness; efficiency; inefficiency; productivity; health service

\section{INTRODUCCIÓN}

El día 2 de noviembre de 2.010, un televidente envió un mensaje a un programa de TV, el cual decía: "Los funcionarios tienen asistencia médica de libre elección (pública o privada), ¿por qué los demás no la tenemos?”.

¿Por qué el modelo sanitario MUFACE (en adelante, modelo MUFACE) tiene tanto éxito (es elegido cada año por más del $80 \%$ de los funcionarios o mutualistas) y es más eficiente con respecto a la producción pública directa? ¿Por qué existen tantos estudios de expertos en Economía Pública sobre la eficiencia, eficacia, efectividad, equidad, sostenibilidad y descentralización de la sanidad y, paradójicamente, se omite $^{3}$ el modelo MUFACE en la práctica totalidad de dichos estudios? ¿Por qué el

\footnotetext{
${ }^{2}$ La gestión privada de los servicios sanitarios públicos de la Comunidad de Madrid ha sido declarada constitucional por el Tribunal Constitucional (Nota informativa núm. 34/2015 del Tribunal Constitucional de 5 de mayo 2015).

${ }^{3}$ Por ejemplo, en Dialnet sólo existen 13 documentos relacionados, directa o indirectamente, con la palabra Muface y, además, la mayoría son de temas jurídicos y prestaciones farmacéuticas.
} 
modelo MUFACE, después de 40 años de contrastado éxito (tanto en eficacia como en eficiencia), no se ha "aplicado" a otros colectivos? ¿Es cierto que, desde hace unos años, los nuevos funcionarios no tienen derecho a elección? ¿Los mutualistas eligen a las empresas privadas cuando son jóvenes y los Servicios Públicos Regionales de Salud de las Comunidades Autónomas (en adelante, SPRS) cuando son mayores?

A todas estas preguntas intentaremos dar respuesta a lo largo del artículo (de forma implícita o explícita).

Asimismo, en los últimos meses se han publicado muchas noticias relacionadas con las Empresas Privadas de Seguros Médicos (en adelante, EPSM) que han suscrito el Concierto con MUFACE para la prestación de los servicios sanitarios a los mutualistas y beneficiarios. Dichas noticias, a modo de resumen, se refieren a que dichas empresas aseguradoras han reducido la oferta de los hospitales en los que pueden recibir los servicios sanitarios los mutualistas y beneficiarios ${ }^{4}$.

\section{BREVE HISTORIA DE MUFACE}

La Mutualidad General de Funcionarios Civiles del Estado (MUFACE) fue creada en 1.975, principalmente, porque a la mayoría de los funcionarios se les prestaban unos servicios sanitarios de inferior calidad respecto al resto de los trabajadores por cuenta ajena. En el momento de la creación, más de 100.000 funcionarios carecían de cobertura contra el riesgo de enfermedad. Se creó MUFACE para resolver o solucionar un problema que tenía un colectivo de aproximadamente 375.000 funcionarios, los cuales no tenían una cobertura de asistencia sanitaria común, e incluso, algunos de ellos, no tenían ningún tipo de cobertura sanitaria. Los funcionarios que tenían cobertura sanitaria, recibían ésta a través de las 65 Mutualidades que existían en los diversos Ministerios. Además, las coberturas sanitarias eran muy diferentes, ya que, mientras algunas Mutualidades prestaban asistencia médico-quirúrgica completa y prestación farmacéutica gratuita, otras sólo cubrían determinadas prestaciones sanitarias. En definitiva, se creó MUFACE para que las prestaciones de los servicios sanitarios fueran iguales para un colectivo de 375.000 funcionarios y 400.000 beneficiarios (personas dependientes del funcionario, como, por ejemplo, el cónyuge e hijos).

\footnotetext{
${ }^{4}$ No obstante, los titulares de diversos periódicos son apocalípticos, aunque, cuando se leen dichos artículos, se trata únicamente de la reducción de la oferta de centros sanitarios (no de la asistencia sanitaria a la que tienen derecho los titulares y beneficiarios o Cartera Común de Servicios del SNS). Dichos titulares, a modo de resumen, dicen: "Un millón de funcionarios ven recortados sus servicios médicos" (http://politica.elpais.com/politica/2015/01/20/actualidad/1421791624_348636.html), "Los beneficiarios de Muface se quedan sin la Oncología y la Radioterapia de MH Hospitales" (http://www.redaccionmedica.com/noticia/los-beneficiarios-de-muface-sequedan-sin-la-oncologia-y-la-radioterapia-de-hm-hospitales-1701) y "Los mutualistas de Isfas y Muface en Madrid no podrán usar HM Hospitales para algunas especialidades" (http://www.europapress.es/madrid/noticia-mutualistas-isfas-muface-madrid-no-podran-usar-hm-hospitales-algunas-especialidades-20150107173256.html).
} 
La Ley 29/1975 facultó a MUFACE para que pudiera prestar los servicios sanitarios directamente (producción y gestión directa) o, alternativamente, a través de Conciertos con empresas públicas o privadas de servicios sanitarios. Dicha Ley manifestaba que los Conciertos "se establecerán preferentemente con Instituciones de la Seguridad Social". Aunque la voluntad del legislador era clara, sin embargo, no se pudo llevar a cabo porque la propia Seguridad Social (el Instituto Nacional de Previsión) no tenía, en aquel momento, la suficiente capacidad para absorber a un colectivo de casi 800.000 personas. En consecuencia, MUFACE tuvo que prestar los primeros servicios sanitarios a través de Conciertos con cuatro EPSM. No obstante, desde 1977, la Seguridad Social (hoy, los SPSR) es una empresa más (además de las empresas privadas) que presta los servicios sanitarios, es decir, los mutualistas, a partir de esa fecha, pueden elegir libremente qué empresa (pública o privada) prefieren que les presten los servicios sanitarios, siendo neutro el coste de dicha elección. Desde dichas fechas, MUFACE suscribe periódicamente Conciertos con diversas EPSM (empresas privadas) al objeto de prestar asistencia sanitaria a los mutualistas y los beneficiarios.

MUFACE, por la experiencia acumulada desde 1975, está especializada en la compra de servicios sanitarios. A través del Concierto (contrato suscrito entre MUFACE y las EPSM), compra para sus mutualistas una cobertura equivalente a la que se presta a través de los SPRS, recibiendo las empresas aseguradoras, como contraprestación, una prima mensual por cada persona asegurada (mutualista o beneficiario). Por tanto, MUFACE sabe con certeza el coste de la prestación de la asistencia sanitaria (per cápita), ya que traslada a las empresas privadas el riesgo de que la prima no cubra el coste real de los servicios que prestan a los mutualistas y beneficiarios de éstos.

El modelo MUFACE posibilita algo esencial: la libertad de elección. Es decir, el mutualista tiene, periódicamente (cada año), el derecho a elegir qué empresa quiere que le preste los servicios sanitarios, lo cual permite alcanzar un elevado nivel de satisfacción. Este modelo sanitario no prejuzga si la sanidad pública es mejor que la privada o viceversa, ya que MUFACE ofrece a sus mutualistas tanto servicios sanitarios producidos por empresas públicas (SPRS) como por empresas privadas (actualmente, Adeslas, DKV, Igualatorio Cantabria y Asisa). Son los mutualistas (no MUFACE) quienes eligen, de acuerdo con sus preferencias y la experiencia propia acumulada, la empresa que le prestara los servicios sanitarios (el coste de la elección es neutro para el mutualista). La libertad de elección, por tanto, se establece entre empresas públicas y privadas y, desde que existe MUFACE, cada año, los mutualistas eligen más del $80 \%(81,75 \%$ en el 2.015$)$ a empresas privadas (EPSM), mientras que menos del 20\% (18,25\% en el 2.015) eligen a empresas públicas (SPRS). Las causas de esta abrumadora preferencia por la producción privada de los servicios sanitarios son dos: la calidad de los servicios sanitarios ofrecidos por las empresas privadas y el alto grado de accesibilidad a dichos 
servicios, ya que no existen listas de espera significativas (en comparación con el sector público). Además, el mutualista no sólo tiene libertad de elegir qué empresa privada le presta los servicios sanitarios, sino que, además, elige qué profesional le presta dichos servicios, entre los muchos que le ofrece dicha empresa privada (EPSM). Finalmente, las EPSM pueden prestar dichos servicios sanitarios a través de: a) medios o infraestructuras propias de las empresas aseguradoras privadas (consultorios, hospitales, etc.), b) contratar a terceros al objeto de que presten los servicios sanitarios (las empresas aseguradoras privadas compiten entre sí para contratar a los proveedores "más eficientes y con la mayor calidad asistencial") y c) una combinación de a y b. Y, por supuesto, los Conciertos que MUFACE suscribe con las empresas aseguradores privadas, no permiten a éstas aplicar períodos de carencia ni realizar selección alguna de riesgos, así como tampoco trasladar los costes de la asistencia a la sanidad pública (Limarquez, M., 2004, pp. 1-5).

Conviene recordar que la asistencia sanitaria en España es universal y gratuita, es decir, el coste de las prestaciones sanitarias se financian íntegramente a cargo del erario público (con los impuestos que pagamos todos los ciudadanos o modelo Beveridge). Es irrelevante el Ministerio que financia la asistencia sanitaria prestada a los ciudadanos a través de los SPRS (Ministerio de Sanidad o CC. AA.) o el que financia la asistencia sanitaria prestada a los mutualistas que eligen a las EPSM (Ministerio de Hacienda). Lo relevante es qué método de producción es más eficiente para el erario público, es decir, cuál es más económico para el conjunto de la sociedad (el bien común).

¿Qué coste soporta MUFACE por los servicios sanitarios que prestan las EPSM a los mutualistas y beneficiarios? ¿Cuál es el coste de la asistencia sanitaria pública (SPRS)? ¿Qué método de producción es más eficiente?

Las partes que suscriben el Concierto son, por una parte, MUFACE y, por otra parte, las EPSM. El objeto de dicho Concierto es la prestación de la asistencia sanitaria a los mutualistas y beneficiarios de MUFACE que opten por recibir dichos servicios sanitarios a través de dichas empresas privadas. Las contingencias cubiertas son las derivadas de enfermedad común o profesional, lesiones derivadas de accidente, cualquiera que sea la causa, incluso si se trata de un acto de terrorismo, por embarazo, parto y puerperio, así como las actuaciones preventivas recogidas en el mismo. La asistencia sanitaria se prestará conforme a la Cartera de Servicios establecida en el Concierto, la cual incluye, cuanto menos, la Cartera Común de Servicios del Sistema Nacional de Salud. Asimismo, las EPSM se obligan a la cobertura de la asistencia sanitaria transfronteriza del colectivo protegido (titulares y beneficiarios) conforme a la Directiva 2011/24/UE del Parlamento y del Consejo Europeo (art. 1.1).

\footnotetext{
${ }^{5}$ Está publicado en el BOE núm. 306, de 23 de diciembre de 2013, disponible a través de: www. muface.es
} 
Dicho Concierto, para el año 2014, estableció una prima fija que se abonó por persona mensualmente y que varió en función de su edad (art. 8.3.1). Si observamos las primas según la pirámide de edad de las personas, la prima menor es para el grupo de edad 5-14 años $(50,91 € /$ mes $)$ y la mayor es para los que tienen más de 74 años $(88,26 € / \mathrm{mes})^{6}$. El importe de las primas que a abonó por MUFACE a las Entidades aseguradoras fue el siguiente:

\begin{tabular}{|c|c|c|}
\hline \multicolumn{3}{|c|}{2014} \\
\hline GRUPOS SEGÚN EDAD & PRIMA MENSUAL & PRIMAS EN BASE 100 \\
\hline $0-4$ & 61,10 & 120,02 \\
\hline $5-14$ & 50,91 & 100,00 \\
\hline $15-44$ & 57,71 & 113,36 \\
\hline $45-54$ & 61,10 & 120,02 \\
\hline $55-64$ & 67,90 & 133,37 \\
\hline $65-74$ & 81,48 & 160,05 \\
\hline Más de 74 & 88,26 & 173,36 \\
\hline
\end{tabular}

Fuente: Concierto de Asistencia Sanitaria 2014.

Asimismo, la prima más elevada (más de 74 años) es un 73,36\% superior a la prima menor (5-14 años), lo cual es lógico como analizaremos posteriormente.

El Concierto para los años 2016 y 2017 contempla un incremento de las primas de un 3,5\% (publicado en el BOE núm. 259 del jueves 29 de Octubre de 2.015).

En el Decreto Ley 13/2010, de 3 de diciembre, el artículo 20 establece que todos los funcionarios que ingresen en cualquier Administración Pública española, a partir del 1 de enero de 2.011, quedan incluidos en el Régimen General de la Seguridad Social, si bien mantendrán, cuando así proceda de acuerdo con su normativa, su cobertura a través del Régimen del Mutualismo Administrativo. En la Exposición de Motivos de dicho Real Decreto Ley se indica que la integración de los funcionarios de nuevo ingreso en el Régimen General de la Seguridad Social lo es a los únicos y exclusivos efectos del Sistema de Clases Pasivas (pensiones de jubilación), manteniéndose para esos funcionarios la acción protectora gestionada en la actualidad por las respectivas Mutualidades de funcionarios. Por tanto, el Real Decreto Ley no cambia el régimen de asistencia sanitaria de las

\footnotetext{
${ }^{6}$ El Concierto para 2015 es similar al del año anterior, excepto la actualización de las primas.
} 
Mutualidades Administrativas (Muface, Isfas y Mugeju), ya que se trata de una modificación que afecta únicamente a las pensiones. Dicho en otras palabras, los nuevos titulares de MUFACE tienen el derecho de optar por recibir la prestación de asistencia sanitaria a través de los SPRS o a través de las EPSM, siendo neutro el coste de dicha elección?.

\section{ANÁLISIS COMPARATIVO DE LOS COSTES DE LOS SPRS CON RESPECTO A MUFACE}

Cuando los poderes públicos deciden que la provisión de un determinado bien o servicio debe ser pública, se debe elegir quién lo producirá (empresa pública o privada) y el criterio único o preferente de elección será la eficiencia económica, o sea, el Estado deberá elegir al productor que le reporte un coste menor por la provisión del bien o servicio público, ya que los recursos públicos son escasos y cualquier ahorro implica menores impuestos o menor endeudamiento o, alternativamente, destinar el ahorro a otras necesidades públicas que no se satisfacen o bien de forma insuficiente.

La producción pública de los bienes y servicios que satisfacen las necesidades públicas, a juicio de Musgrave (1969, p. 48), sólo está justificada en aquellas situaciones en las que la eficiencia económica de la empresa pública es superior a la producción privada bajo control público. Dicho en otras palabras, la producción de un bien o servicio público, lógicamente, la realizará la empresa que lo haga al menor coste posible, es decir, la empresa que sea más eficiente en la asignación de recursos (Rosen, 2002, p. 68), ya que, “... se trata de un proceso técnico y, por tanto, debe producirlos el agente económico, público o privado, que sea más eficiente" (Barea y Gómez, 1999, p. 78). En definitiva, los bienes preferentes (sanidad, educación, etc.) los deben producir las empresas (públicas o privadas) que lo hagan al menor coste posible para el Estado, sin obviar la calidad mínima exigible en la producción de cualquier bien preferente. La calidad que el Estado exige en la producción de un bien preferente a la empresa productora (pública o privada) se puede regular en un contrato, en el cual se especifiquen los controles o supervisiones que debe realizar el Estado (López Saludas, JM., 2013a, pp. 173-193).

La eficiencia y la productividad no son conceptos sinónimos pero están interrelacionados. Si una empresa incrementa la productividad, ello significa que se obtiene mayor cantidad de producto con el mismo consumo de recursos o factores

\footnotetext{
${ }^{7}$ En los últimos dos años se han publicado noticias y comentarios contradictorios en internet con respecto a si los nuevos funcionarios tienen derecho a elección. Se solicitó información a MUFACE al objeto de aclarar algunos aspectos. Se ha transcrito, de forma casi literal, la amable respuesta que se recibió de la Secretaría General de MUFACE. Desde estas líneas se agradece muy sinceramente la disponibilidad de la Mutualidad y dicha respuesta.
} 
de producción, o bien obtener la misma cantidad de producción con menores costes. Aunque en la mayoría de los casos, el incremento de la productividad de la mano de obra repercute positivamente en la eficiencia económica de la empresa (reducción de los costes unitarios), no obstante, a veces, "una empresa puede haber alcanzado una elevada productividad de la mano de obra a costa de un despilfarro de las materias primas o de la excesiva utilización del equipo y maquinaria, con lo cual el resultado neto para la empresa podría ser desfavorable" (Fernández Pirla, 1974, pp. 267-275). En consecuencia, cuando se pretende conseguir que una empresa (pública o privada) aumente su eficiencia económica, sin modificar la tecnología existente, la vía principal es aumentar la productividad ${ }^{8}$ de todos los factores productivos que se precisan para producir los bienes y servicios.

La diferencia de la eficiencia económica del sector público con respecto al sector privado, como veremos a continuación, es significativa y, en gran medida, es atribuible a las diferencias salariales entre ambos sectores. En el período 20052012, la diferencia fue del $36 \%{ }^{9}$, es decir, los salarios medios en el sector público fueron un 36\% más elevados que en el sector privado, aunque si se tienen en consideración "las distintas características observables" (edad, antigüedad, grupos de cotización, tipo de contrato, tamaño de la empresa, etc.) dicha diferencia se reduce al 20,5\% (Hospido y Moral-Benito, 2015, pp. 19-25).

Según PriceWaterhouseCoopers (2010, pp. 10-11), en el período 1999-2004, el diferencial entre el gasto sanitario público per cápita (producción pública) por prestar la asistencia sanitaria fue, en promedio, un $50 \%$ más caro que la prima per cápita que percibieron las EPSM de MUFACE por prestar el mismo servicio sanitario a los mutualistas y beneficiarios. En definitiva, según este estudio, el coste de la prestación de los mismos servicios sanitarios a través de los SPRS (producción pública) fue el $50 \%$ más caro (ineficiencia) con respecto a la prima per cápita que pagó MUFACE a las EPSM (producción privada).

Respecto al período 2005-2010, el coste sanitario total devengado por los SPRS con respecto al coste total del modelo MUFACE (ambos incluyen la asistencia sanitaria, la farmacia, prótesis, traslados de enfermos y otras prestaciones), la ineficiencia de las CC. AA. en la producción de los mismos servicios sanitarios fue entre el $55 \%$ y $60 \%$, la cual no difiere del estudio realizado por la Consultora PriceWaterhouseCoopers. Y si la comparación es únicamente entre el coste de la asistencia sanitaria (no incluye la farmacia, prótesis y otras prestaciones), las CC. AA. incurrieron en un "exceso de coste" (o ineficiencia) respecto al modelo MUFACE de 419,56€ per cápita (año 2009), lo cual, en porcentaje representa el

${ }^{8}$ A veces, las mejoras de la productividad se consiguen a través de la disminución de la eficacia (calidad del servicio).

${ }^{9}$ Boletín Mensual Banco España de Febrero 2015.

http://www.bde.es/f/webbde/SES/Secciones/Publicaciones/InformesBoletinesRevistas/BoletinEconomico/15/Feb/Fich/be1502-art3.pdf 
60,04 \%. Dicho en otras palabras, el coste de producción (per cápita) de los servicios sanitarios (homogéneos) del sector público es considerablemente superior al sector privado (López Saludas, 2013c, pp. 173-193).

\begin{tabular}{|l|r|r|r|}
\cline { 2 - 4 } \multicolumn{1}{l|}{} & \multicolumn{1}{c|}{2011} & \multicolumn{1}{c|}{2012} & \multicolumn{1}{c|}{2013} \\
\hline Coste sanitario público total (1) & 68.050 & 64.153 & 61.710 \\
\hline $\begin{array}{l}\text { Coste total de la Mutualidad } \\
\text { de Funcionarios (1) }\end{array}$ & 2.085 & 2.094 & 2.131 \\
\hline $\begin{array}{l}\text { Coste sanitario total de las Comunidades } \\
\text { Autónomas + Ceuta y Melilla (1) }\end{array}$ & 62.609 & 59.109 & 56.761 \\
\hline Población total en España & 47.190 .493 & 47.265 .321 & 47.129 .783 \\
\hline $\begin{array}{l}\text { Mutualistas (Muface+Isfas+Megeju) } \\
\text { que eligen empresas privadas de seguro } \\
\text { médico (3) }\end{array}$ & 1.971 .362 & 1.960 .267 & 1.951 .098 \\
\hline $\begin{array}{l}\text { Población total protegida por las } \\
\text { Comunidades Autónomas (SPRS) }\end{array}$ & 45.219 .131 & 45.305 .054 & 45.178 .685 \\
\hline $\begin{array}{l}\text { Coste total público per cápita de los SPRS } \\
\text { de las CC. AA (en euros) }\end{array}$ & $1.384,57$ & $1.304,69$ & $1.256,37$ \\
\hline $\begin{array}{l}\text { Coste total público de los mutualistas } \\
\text { de Muface que eligen EPSM (4) }\end{array}$ & $1.225,18$ & $1.242,86$ & $1.272,67$ \\
\hline $\begin{array}{l}\text { Total de Titulares y Beneficiarios de } \\
\text { MUFACE que eligen empresas privadas }\end{array}$ & 1.266 .693 & 1.257 .449 & 1.249 .177 \\
\hline $\begin{array}{l}\text { Coste total per cápita de los mutualistas } \\
\text { que eligen sanidad privada (en euros) }\end{array}$ & 967,22 & 988,40 & $1.018,81$ \\
\hline Diferencial o exceso de coste sector público & 417,35 & 316,29 & 237,56 \\
\hline Ineficiencia del sector público & $43,15 \%$ & $32,00 \%$ & $23,32 \%$ \\
\hline
\end{tabular}

(1) Datos del Ministerio de Sanidad (en millones de euros).

(2) Datos publicados por el INE.

(3) Datos de ICEA a 31 de diciembre de cada año.

(4) Datos obtenidos de las memorias de Muface 2011, 2012 y 2013 (en millones de euros). Fuente: Elaboración propia utilizando datos del Ministerio de Sanidad y Memorias Muface a fecha 27/5/15.

Respecto a los últimos datos publicados por el Ministerio de Sanidad (años 2011, 2012 y 2013), se produjo una disminución considerable del coste sanitario total de los SPRS (Comunidades Autónomas), el cual fue 9,34\% ${ }^{10}$. Dicha

$$
\left.{ }^{10}((62.609-56.761) / 62.609)\right)=9,34 \%
$$


disminución es la causa principal de la reducción de la ineficiencia del sector público con respecto al modelo MUFACE (desde el 43,15\% al 23,32\%), aunque también contribuyó la disminución de la población protegida ( 40.446 personas), sin perjuicio del posible incremento de la productividad. Pero esa ineficiencia es aparente, ya que la "real" es mucho mayor. ¿Por qué es superior dicha ineficiencia?

Si bien es cierto que la práctica totalidad de los ciudadanos que residen en España tienen derecho a la asistencia sanitaria gratuita a través de los SPRS, no obstante, un segmento considerable tiene "doble cobertura". La "doble cobertura" consiste en que determinados usuarios ${ }^{11}$ de los SPRS, por las razones que sean, suscriben un contrato privado con las EPSM para que éstas les presten determinados servicios sanitarios (atención primaria, especializada, urgencias, intervenciones quirúrgicas, etc.). Generalmente, dichos contratos o pólizas consisten en que el asegurado paga una prima mensual y, como contraprestación, aquél tiene libertad para elegir los profesionales sanitarios y hospitales que ofrece la empresa aseguradora. Por tanto, lo lógico es que las personas que suscriben dichos contratos médicos privados sean las personas a las que obligatoriamente se las adscriben a los SPRS y que, por lo motivos que sean, no están satisfechos con los servicios sanitarios públicos que reciben (según el Barómetro Sanitario de 2013, el 34\% no está satisfecho con el funcionamiento de los servicios sanitarios públicos, ya que consideran que "necesita cambios fundamentales" y "habría que rehacerlo"). Hay que recordar, una vez más, que unos determinados colectivos de funcionarios (MUFACE, ISFAS y MUGEJU) tienen "derecho de opción"12, es decir, cada año, de acuerdo con sus experiencias previas, eligen si el proveedor que les prestará los servicios sanitarios será el sector público (los SPRS) o el sector privado (las EPSM). ¿Por qué motivo un mutualista que elige una EPSM (a coste cero) debería suscribir (pagar) otro seguro privado con la misma u otra EPSM?

En relación a la información de Investigación Cooperativa entre Entidades Aseguradoras (ICEA), en el año 2014, 6.385 .912 personas tuvieron doble cobertura ${ }^{13}$ y las primas que pagaron ascendieron a 4.745 millones de euros por los diversos contratos de seguros médicos privados que suscribieron con las diversas empresas aseguradoras privadas, lo cual supone que la prima media anual por

${ }^{11}$ La web de DKV decía: "el mercado del aseguramiento sanitario privado está dominado por los seguros de asistencia sanitaria. Se denomina de doble cobertura porque casi todos nuestros asegurados privados tienen una cobertura sanitaria pública en el Sistema Nacional de Salud.

(http://www.dkvseguros.com/newsletterdkv/documentos/revistas/interior311_62.htm).

${ }^{12}$ El "derecho de opción" (o libertad de elección) consiste en que los usuarios de los servicios sanitarios (asistencia sanitaria) eligen quién será el proveedor de dichos servicios: las empresas públicas o las empresas privadas. Pueden elegir el cuadro médico y hospitalario que la EPSM ofrece en toda España, sin copago ni límites de visitas, salvo algunas excepciones (por ejemplo, una limpieza bucal por año).

${ }^{13}$ No se incluye otras 672.120 personas que pagaron 642 millones en concepto de reembolso de gastos sanitarios, lo cual supone una prima per cápita de $955 €$. 
persona asegurada fue de 606,27 euros ${ }^{14}$. Lógicamente, estos 6,4 millones de personas "descargan" a la sanidad pública (SPRS), es decir, si estas personas no se costearan un seguro médico privado, los SPRS deberían prestar los servicios que aquéllas reciben a través de sus seguros médicos privados y, por tanto, el coste de la sanidad pública se incrementaría, como mínimo ${ }^{15}$, en el valor de las primas que pagan a las empresas aseguradoras.

Como indican Abásolo, Pinilla y Negrin (2008, p. 102), la "doble cobertura" significa un menor uso de todos los servicios sanitarios públicos, y, por tanto, "este hecho sugiere que el seguro privado es de facto un sustituto de los servicios sanitarios públicos". Por consiguiente, hay que cuantificar el efecto económico del "no uso" de los servicios sanitarios públicos, es decir, se trata de estimar el verdadero gasto sanitario público per cápita teniendo en consideración el elevado número de usuarios de los SPRS que, teniendo derecho a utilizar dichos servicios, de hecho, no los utilizan o lo hacen muy poco. Asimismo, también existe otro tipo de usuarios que también "descargan" o "alivian" a los SPRS: aquellos usuarios que no tienen un seguro médico privado pero que, sin embargo, pagan a los proveedores privados por cada "acto médico" (operaciones, visitas a profesionales, etc.). O sea, se trata de unas personas que tienen derecho a la asistencia sanitaria pública que recurren a la asistencia sanitaria privada (por ejemplo, porque no aceptan los días que se debe esperar para ser intervenidos quirúrgicamente, o bien no están satisfechos con los servicios recibidos por los SPRS, o porque no pueden elegir al profesional médico que prefieren que les asistan, etc.).

Según el "Sistema de Cuentas de Salud" (SCS), en el año 2012 16 , el gasto privado sanitario ascendió a 27.064 millones de euros, el cual fue financiado a través de:

a) seguros privados suscritos con las empresas aseguradoras (5.494 millones de euros, el 20,30\% ${ }^{17}$ ),

b) "pagos directos de los hogares" (20.865 millones de euros, el 77,10\% $\left.{ }^{18}\right)$ y,

c) el resto (705 millones de euros, 2,60\%) a través de "instituciones sin fines de lucro al servicio de los hogares" y "sociedades".

Como se puede constatar, la mayoría del gasto sanitario privado se financia a través de "pagos directos de los hogares" que realizan las personas cuando visitan

${ }^{14}$ 5.096,30 millones de $€ / 8.405 .976=606,27 €$.

${ }^{15}$ Se supone que el sector público presta los servicios sanitarios con la misma eficiencia que el sector privado.

${ }^{16}$ Metodología que aplica la OCDE-EUROSTAT-OMS para calcular el Gasto Sanitario Total. Los datos que a continuación se analizan están disponibles en la web del Ministerio de Sanidad, Servicios Sociales e Igualdad. Los datos del año 2012 son los últimos datos publicado a fecha 23/5/2015.

${ }^{17}(5.494 / 27.064) \times 100=20,30 \%$

${ }^{18}(20.865 / 27.064) \times 100=77,10 \%$ 
a un médico privado, se operan en un hospital privado, etc. Si se analiza con mayor detalle en el año 2012, dicho concepto se desglosa en:

a) 574 millones de euros $(2,75 \%)$ de "servicios de asistencia curativa y de rehabilitación hospitalaria",

b) 9.098 millones de euros $(43,60 \%)$ a "asistencia curativa y de rehabilitación ambulatoria" 19

c) 3.561 millones de euros $(17,07 \%)$ a "servicios de atención de larga duración"20,

d) 91 millones de euros (0,44\%) a "servicios auxiliares de atención a la salud" y,

e) 7.542 millones de euros $(36,15 \%)$ a "productos médicos dispensados a pacientes ambulatorios"21.

Si la sanidad pública (SPRS) funcionara óptimamente o mejor que en la actualidad (por ejemplo, que las listas de espera fueran casi inexistentes, que hubiera derecho o libertad de elección de médicos y centros hospitalarios, etc.), lógicamente, la mayoría de los 6,4 millones de personas que antes se comentaban no contratarían seguros médicos privados ni, asimismo, muchas personas recurrirían a la medicina privada si no tuvieran que esperar tanto tiempo para ser atendidos ("pagos directos de los hogares", según el SCS). En consecuencia, si la sanidad es un bien preferente de carácter universal y gratuito, tal y como está configurado, el verdadero o auténtico coste sanitario público (SPRS) es la suma de la financiación pública (coste devengado por los SPRS) y una parte importante de la financiación privada ya que la mayoría de los 6,4 millones de personas que suscriben los seguros médicos privados, así como, los que pagan los servicios médicos privados son usuarios de los SPRS que, por diversos motivos, no utilizan o lo hacen parcialmente los servicios que presta la sanidad pública. Dicho en otras palabras, "descongestionan" los SPRS y ello es un ahorro, ya que si nadie contratara seguros médicos privados o nadie recurriera a la medicina privada, las listas de espera serían mayores (disminuiría la eficacia) o bien se precisarían de más recursos materiales y humanos (aumento del coste total de los SRPS). Por consiguiente, el "verdadero" coste sanitario público es la suma resultante del coste de lo que "ha hecho" más el coste de lo que "debería haber hecho". Esta metodología es similar a la que aplica la empresa privada para calcular los costes históricos (reales) unitarios que ha incurrido en la producción de un bien o

\footnotetext{
${ }^{19}$ Se desglosan en: a) asistencia odontológica ambulatoria (5.229 millones de $€$ ), b) servicios de asistencia especializada (2.797 millones de $€$ ), c) otros servicios de asistencia ambulatoria (1.071 millones de $€)$.

${ }^{20}$ Se desglosan en: a) atención de larga duración hospitalaria (2.518 millones de €), b) atención de larga duración en hospitalización de día (319 millones de €), c) atención de larga duración domiciliaria (723 millones de €).

${ }^{21}$ Se desglosan en: a) productos farmacéuticos y otros productos médicos perecederos (5.042 millones de $€$ ) y b) dispositivos terapéuticos y otros productos médicos no perecederos (2.500 millones de $€$ ).
} 
servicio. Aplicando esta metodología, los costes sanitarios públicos per cápita fueron $1.654,24 €, 1.627,93 €$ y $1.590,02 €$, en los años 2009 , 2010 y 2011 , respectivamente, lo cual significó una ineficiencia con respecto al modelo MUFACE del 75,66\% 71,16\% y 64,39\% respectivamente (López Saludas, 2013c, pp. 69-70).

Asimismo, si hacemos la comparación, únicamente, entre el coste de la asistencia sanitaria $^{22}$ de las Comunidades Autónomas (SPRS) y el modelo MUFACE, las primeras incurrieron en un exceso de coste, o ineficiencia, respecto al modelo MUFACE de 482,75€, 399,29€ y 332,78€ per cápita en los años 2011, 2012 y 2013 lo cual, en porcentaje, representa el $63,10 \%$, el 51,33\% y el $41,24 \%$ \% de ineficiencia respectivamente.

\begin{tabular}{|l|r|r|r|}
\cline { 2 - 4 } \multicolumn{1}{l|}{} & \multicolumn{1}{c|}{2011} & \multicolumn{1}{c|}{2012} & \multicolumn{1}{c|}{2013} \\
\hline $\begin{array}{l}\text { Coste sanitario total de las Comunidades } \\
\text { Autónomas (1) }\end{array}$ & 62.609 & 59.109 & 56.761 \\
\hline Población total en España (2) & 47.190 .493 & 47.265 .321 & 47.129 .783 \\
\hline $\begin{array}{l}\text { Población total protegida por las Comunida- } \\
\text { des Autónomas (SPRS) }\end{array}$ & 45.219 .131 & 45.305 .054 & 45.178 .685 \\
\hline $\begin{array}{l}\text { Particulares que suscriben seguros de asisten- } \\
\text { cia sanitaria }\end{array}$ & 6.006 .788 & 6.114 .842 & 6.261 .047 \\
\hline $\begin{array}{l}\text { Población "real" protegida por las Comunida- } \\
\text { des Autónomas (hipótesis) }\end{array}$ & 39.212 .343 & 39.190 .212 & 38.917 .638 \\
\hline $\begin{array}{l}\text { Coste de los servicios sanitarios de los SPRS } \\
\text { (1) y (2) }\end{array}$ & 48.929 & 46.194 & 44.359 \\
\hline $\begin{array}{l}\text { Coste de los servicios per cápita "real" de los } \\
\text { servicios sanitarios públicos (3) }\end{array}$ & $1.247,79$ & $1.178,70$ & $1.139,81$ \\
\hline $\begin{array}{l}\text { Coste de la asistencia sanitaria de los mutua- } \\
\text { listas que eligen empresas privadas (1) y (4) }\end{array}$ & 969,07 & 979,43 & $1.008,12$ \\
\hline $\begin{array}{l}\text { Total de Titulares y Beneficiarios. de } \\
\text { MUFACE que eligen Empresas Privadas }\end{array}$ & 1.266 .693 & 1.257 .449 & 1.249 .177 \\
\hline $\begin{array}{l}\text { Coste per cápita de la asistencia sanitaria de } \\
\text { los mutualistas que eligen empresas privadas }\end{array}$ & 765,04 & 778,90 & 807,03 \\
\hline Diferencial o exceso de coste sector público & 482,75 & 399,80 & 332,78 \\
\hline Ineficiencia del sector público & $63,10 \%$ & $51,33 \%$ & $41,24 \%$ \\
\hline
\end{tabular}

(1) en millones de euros.

(2) Se excluyen: farmacia, traslado, prótesis, aparatos terapéuticos, servicios de salud pública y docencia MIR (son, aprox., un 78,15\% del total).

(3) Se refiere a los servicios hospitalarios, especializados y primarios de salud.

(4) Memorias Muface 2011, 2012 y 2013 (apartado “ejecución presupuestaria”).

\footnotetext{
${ }^{22}$ Se excluye la farmacia, traslado, prótesis, aparatos terapéuticos, servicios de salud pública y docencia MIR.
} 
Si añadiéramos una parte de los "pagos directos" (los servicios hospitalarios y ambulatorios), así como los seguros que se pagan por "reembolsos de gastos" (sanitarios), el diferencial o exceso de coste del sector público aún sería mayor.

En definitiva, podemos afirmar que el modelo MUFACE es mucho más eficiente que el modelo imperante en los Servicios Regionales de Salud (Comunidades Autónomas.), el cual se caracteriza por ser, mayoritariamente, un modelo de producción pública y gestión directa de los servicios sanitarios. La ineficiencia del sector público con respecto al modelo MUFACE (sector privado) implica que aquél produce los mismos servicios a un coste superior que éste (entre el $40 \%$ y el 60\%). Este exceso de coste (ineficiencia) del sector público con respecto al privado ha sido permanente a lo largo de los períodos analizados (1999 - 2013).

\section{ANÁliSiS DEL COLECTIVO MUFACE}

El colectivo de los mutualistas en activo de MUFACE es muy heterogéneo tal y como se puede constatar pero, sin embargo, el colectivo preponderante es el que se refiere a los docentes, los cuales representan el $65,44 \%{ }^{23}$ del total. Asimismo, el colectivo de mutualistas en activo representa el $67,55 \%$ de los mutualistas o titulares.

\begin{tabular}{|l|r|r|}
\hline \multicolumn{2}{|c|}{2013} \\
\hline $\begin{array}{l}\text { COLECTIVO DE MUTUALISTAS EN ACTIVO O } \\
\text { ASIMILADO }\end{array}$ & NÚMERO & \multicolumn{1}{c|}{$\%$} \\
\hline INSPECTORES Y GESTIÓN DE HACIENDA & 6.809 & $1,05 \%$ \\
\hline PROFESORES UNIVERSIDAD & 47.028 & $7,22 \%$ \\
\hline PROFESORES ENSEÑANZA SECUNDARIA & 147.747 & $22,69 \%$ \\
\hline $\begin{array}{l}\text { MAESTROS Y PROFESORES TECNICOS DE FORMA- } \\
\text { CIÓN PROFESIONAL }\end{array}$ & 225.695 & $34,67 \%$ \\
\hline OTROS PROFESORES & 5.570 & $0,86 \%$ \\
\hline MEDICOS, FARMACÉUTICOS Y VETERINARIOS & 3.058 & $0,47 \%$ \\
\hline POLICIAS & 80.952 & $12,43 \%$ \\
\hline POSTALES Y TELECOMUNICACIONES & 24.929 & $3,83 \%$ \\
\hline ADMINISTRATIVOS DEL ESTADO & 49.194 & $7,56 \%$ \\
\hline INSTITUCIONES PENITENCIARIAS & 21.542 & $3,31 \%$ \\
\hline OTROS COLECTIVOS & 38.493 & $5,91 \%$ \\
\hline TOTAL & 651.017 & $100,00 \%$ \\
\hline
\end{tabular}

Fuente: elaboración propia con la Memoria Muface 2013.

\footnotetext{
${ }^{23}((47.028+147.747+225.695+5.570) / 651.017) \times 100=65,44 \%$.
} 
Por lo que respecta a los mutualistas jubilados, éstos son el 27,84\% del total del colectivo; el resto $(4,61 \%)$, son "beneficiarios con documento asimilado al de afiliación y mutualistas voluntarios" ${ }^{24}$.

Desde que se creó o instauró el modelo MUFACE, los mutualistas año tras año, cuando ejercitan el derecho de elección, lo hacen abrumadoramente por recibir la asistencia sanitaria a través de las EPSM (entre el 82\% y el 85\%, aproximadamente); no obstante, desde el año 2007 se puede constatar una ligera disminución (desde el 85,48\% al 81,79\%).

\begin{tabular}{|l|c|c|c|c|c|c|c|c|}
\cline { 2 - 8 } \multicolumn{1}{c|}{} & \multicolumn{7}{c|}{ DISTRIBUCIÓN DEL COLECTIVO ASEGURADO EL 31 DE } \\
\cline { 2 - 8 } & 2007 & 2008 & 2009 & 2010 & 2011 & 2012 & 2013 & 2014 \\
\hline $\begin{array}{l}\text { PICIEMBRE } \\
\text { Titulares y } \\
\text { Beneficiarios que } \\
\text { eligen el sector } \\
\text { privado (Adeslas, } \\
\text { Asisa, DKV, etc.) }\end{array}$ & $85,48 \%$ & $84,55 \%$ & $83,84 \%$ & $82,81 \%$ & $82,23 \%$ & $81,92 \%$ & $81,88 \%$ & $81,83 \%$ \\
\hline $\begin{array}{l}\text { Porcentaje de } \\
\text { Titulares y } \\
\text { Beneficiarios que } \\
\text { eligen el sector } \\
\text { público (SPRS) }\end{array}$ & $14,52 \%$ & $15,45 \%$ & $16,16 \%$ & $17,16 \%$ & $17,76 \%$ & $18,08 \%$ & $18,11 \%$ & $18,17 \%$ \\
\hline
\end{tabular}

Fuente: elaboración propia con Memorias Muface y datos facilitados por Muface.

En el mes de enero de 2015, los mutualistas eligieron un porcentaje muy similar al de los años precedentes, es decir, el $81,32 \%{ }^{25}$ de los mutualistas optaron por recibir la asistencia sanitaria a través de las EPSM.

Si analizamos los tramos de edad del colectivo de mutualistas y beneficiarios se constata un progresivo envejecimiento ${ }^{26}$ del tramo de 50 a 74 años (del 33,57\% al $39,03 \%$ ) y a partir de los 75 años (del 5,96\% al 6,33\%).

\footnotetext{
${ }^{24}$ Memoria MUFACE 2013, p. 63.

${ }^{25}$ Según elEconomista.es, el colectivo de mutualistas y beneficiarios eligieron recibir la asistencia sanitaria siguiente: Adeslas (549.876), Asisa (455.148), Igualatorio Cantabria (7.291), DKV (215.621) y los SPRS (282.039). Los SPRS representan el $18,68 \%((282.039 / 1.509 .975)$ x 100$)$ y el resto $(81,32 \%)$ son las EPSM (publicado el 16/03/2015).

${ }^{26}$ Los datos de 2011, 2012 y 2013 confirman el progresivo envejecimiento en los tramos citados. De nuevo, agradezco a la Secretaría General de Muface por proporcionarme dicha información, la cual no consta en ninguna Memoria ni, que yo sepa, en ningún artículo publicado. Dichos datos están más depurados que los publicados en las Memorias (datos a 31 de diciembre de cada año), ya que desde el año 2011 se han introducido nuevas herramientas informáticas del control en la gestión del colectivo (datos homogéneos del período 2010 a 2014).
} 


\section{ELECCIÓN DE MUTUALISTAS Y BENEFICIARIOS SEGÚN LOS TRAMOS DE EDAD}

\begin{tabular}{|c|c|c|c|c|c|c|c|c|c|c|c|c|}
\hline \multirow[b]{3}{*}{ EDAD } & \multicolumn{6}{|c|}{ AÑO 2010} & \multicolumn{6}{|c|}{ AÑO 2014} \\
\hline & \multicolumn{2}{|c|}{ SPSR } & \multicolumn{2}{|c|}{ EPSM } & \multicolumn{2}{|c|}{ TOTAL } & \multicolumn{2}{|c|}{ SPSR } & \multicolumn{2}{|c|}{ EPSM } & \multicolumn{2}{|c|}{ TOTAL } \\
\hline & $\begin{array}{c}\text { PERSO- } \\
\text { NAS }\end{array}$ & $\%$ & \begin{tabular}{|c|} 
PERSO- \\
NAS \\
\end{tabular} & $\%$ & $\begin{array}{c}\text { PERSO- } \\
\text { NAS } \\
\end{array}$ & $\%$ & $\begin{array}{l}\text { PERSO- } \\
\text { NAS }\end{array}$ & $\%$ & \begin{tabular}{|c|} 
PERSO- \\
NAS \\
\end{tabular} & $\%$ & \begin{tabular}{|c|} 
PERSO- \\
NAS \\
\end{tabular} & $\%$ \\
\hline 00 a 25 & 81.342 & 18,71 & 353.415 & 81,29 & 434.757 & 27,92 & 82.969 & 20,09 & 330.017 & 79,91 & 412.986 & 27,22 \\
\hline 25 a 49 & 95.789 & 18,90 & 411.013 & 81,10 & 506.802 & 32,55 & 78.169 & 18,79 & 337.827 & 81,21 & 415.996 & 27,42 \\
\hline 50 a 74 & 78.950 & 15,10 & 443.766 & 84,90 & 522.716 & 33,57 & 106.646 & 18,01 & 485.359 & 81,99 & 592.005 & 39,03 \\
\hline 75 a 99 & 10.227 & 11,12 & 81.743 & 88,88 & 91.970 & 5,91 & 7.787 & 8,17 & 87.473 & 91,83 & 95.260 & 6,28 \\
\hline más de 99 & 97 & 12,63 & 671 & 87,37 & 768 & 0,05 & 87 & 12,10 & 632 & 87,90 & 719 & 0,05 \\
\hline TOTAL & 266.405 & 17,11 & 1.290 .608 & 82,89 & 1.557 .013 & 100,00 & 275.658 & 18,17 & 1.241 .308 & 81,83 & 1.516 .966 & 100,00 \\
\hline
\end{tabular}

Fuente: elaboración propia a partir de los datos facilitados por MUFACE.

Según el Informe del Grupo de Trabajo sobre Gasto Sanitario de la Intervención General de la Administración General del Estado (2005), una persona mayor de 75 años consume 3,12 veces la media del gasto hospitalario por cápita. Asimismo, las personas de edades comprendidas entre los 65 y 74 años y las mayores de 75 años consumen 3,21 y 4,30 veces la media del gasto farmacéutico por cápita.

Un mito muy propagado ${ }^{27}$ es algo parecido a lo siguiente: cuando el mutualista es joven elige a las empresas privadas (EPSM) y cuando es anciano elige lo público (los SPRS). Pues bien, ese mito no se corresponde con la realidad ya que, como se puede constatar en la siguiente tabla, es todo lo contrario, es decir, a partir de los 80 años el porcentaje de los que eligen a las EPSM (producción privada) oscila entre el $86 \%$ y el $88 \%$, cuyo porcentaje es superior a la media ( $82 \%$ a $85 \%$ ). Además, conviene recordarlo, el Concierto no permite la selección de riesgos, ya que la decisión de "salir y entrar" en cualquiera de las EPSM corresponde exclusivamente al mutualista.

En resumen, las EPSM no seleccionan el riesgo, o sea, es una gran mentira el siguiente mantra o mito: las empresas aseguradoras privadas seleccionan a las personas jóvenes y sanas y la sanidad pública la eligen las mayores y enfermas. Y, además, desde hace muchos años, la edad media del colectivo MUFACE que elige a las empresas privadas es superior a los que eligen la sanidad pública (Razquin, PM, 1998, pp. 259-275).

${ }^{27}$ En cualquier discusión informal entre amigos y opiniones en internet. Por ejemplo, sirva de ejemplo el siguiente comentario en internet: "Falta destacar que las aseguradoras escogen sus pacientes. No se trata de un descremado sociológico. No aseguran pacientes crónicos, ni personas con secuelas de accidentes, ni con historias clínicas con alguna complicación..... Norma. Porque, al final, las mutualidades y aseguradoras se convierten en empresas financieras con respaldo estatal". 


\begin{tabular}{|c|c|c|c|c|c|c|}
\cline { 2 - 7 } \multicolumn{1}{c|}{} & \multicolumn{5}{c|}{ AÑO 2014} \\
\hline \multirow{2}{*}{$\begin{array}{c}\text { TRAMOS } \\
\text { DE EDAD }\end{array}$} & \multicolumn{2}{|c|}{ SPSR } & \multicolumn{2}{c|}{ EPSM } & \multicolumn{2}{c|}{ TOTAL } \\
\cline { 2 - 8 } & PERSONAS & $\%$ & PERSONAS & $\%$ & PERSONAS & $\%$ \\
\hline 80 a 84 & 3.563 & $12,07 \%$ & 25.954 & $87,93 \%$ & 29.517 & $47,96 \%$ \\
\hline 85 a 89 & 2.552 & $13,41 \%$ & 16.478 & $86,59 \%$ & 19.030 & $30,92 \%$ \\
\hline 90 a 94 & 1.265 & $13,42 \%$ & 8.159 & $86,58 \%$ & 9.424 & $15,31 \%$ \\
\hline 95 a 99 & 407 & $14,27 \%$ & 2.446 & $85,73 \%$ & 2.853 & $4,64 \%$ \\
\hline más de 99 & 87 & $12,10 \%$ & 632 & $87,90 \%$ & 719 & $1,17 \%$ \\
\hline TOTAL & 7.874 & $12,79 \%$ & 53.669 & $87,21 \%$ & 61.543 & $100,00 \%$ \\
\hline
\end{tabular}

Fuente: elaboración propia a partir de los datos facilitados por MUFACE.

Respecto a la medición de la eficacia, el indicador "grado o índice de satisfacción del cliente" es el que más se utiliza para medir la calidad de los diversos servicios públicos (por ejemplo, servicios sanitarios y educativos). Dicho indicador mide la percepción que tienen los clientes ${ }^{28}$ de un determinado servicio público. La calidad del servicio percibido por el cliente será más alta cuanto mayor sea la diferencia entre la percepción del servicio recibido en comparación con las expectativas que tenía antes de recibirlo. Así, si las expectativas del cliente o usuario son muy altas y el servicio recibido no las supera, percibirá que el servicio no tiene la calidad que el cliente esperaba. Dicho en otras palabras: la calidad del servicio que percibe el cliente o usuario es la relación existente entre el servicio recibido (percibido) y el servicio esperado (expectativas). Cualquier Administración Pública que preste un servicio público (especialmente, los bienes preferentes), deberá tener como objetivo estratégico que los clientes reciban (perciban) un servicio mejor que el que esperaban recibir. En definitiva, no sólo el servicio debe ser de calidad sino que además tiene que aparentar dicha calidad. Para lograr ese objetivo, las personas que prestan dicho servicio son el principal elemento o ventaja competitiva.

El grado de satisfacción de los usuarios de la sanidad pública es buena o aceptable $(6,59,6,57$ y 6,41 en $2.011,2012$ y 2.013 , respectivamente), sin embargo, no funciona ni con la deseable eficacia (muchos usuarios recurren a la sanidad privada), ya que cuando las personas tienen el "derecho de elección" entre la sanidad producida por el sector público o por el sector privado, lo hacen mayoritariamente por este último (año tras año lo hacen más del 80\% de los mutualistas).

\footnotetext{
${ }^{28}$ El término "cliente" de un servicio público se utiliza cada vez más en detrimento de "usuario".
} 
En definitiva, el verdadero grado de satisfacción se manifiesta cuando las personas que tienen libertad de elección entre la producción pública (SPRS) y la privada (modelo MUFACE o similar) de los servicios sanitarios públicos, sin embargo, sólo una pequeña parte (15-18\%) eligen la producción pública. La eficacia del sector público mejoraría si compitiera, en igualdad de condiciones, con el sector privado, lo cual presupone que los ciudadanos tienen libertad de elección (a coste neutro). ¿Acaso este dato objetivo no es una constatación o evidencia de que la eficacia es mayor en el sector privado?

\section{5. ¿EL MODELO SANITARIO DE MUFACE ES APLICABLE A OTROS COLECTIVOS DE FUNCIONARIOS O TRABAJADORES POR CUENTA AJENA?}

En opinión del autor del presente artículo, no hay ningún impedimento para que, a través de un proceso paulatino, se incorporen nuevos colectivos ya que, como se ha indicado, el modelo MUFACE es eficaz (alto grado de satisfacción de los usuarios) y contribuye a la viabilidad económica del SNS porque es mucho más eficiente que el modelo publico imperante en los SPRS (un 40\%-60\%) en lo que se refiere a la asistencia sanitaria, Como dijo Dña. Alicia Sánchez Gil, Directora Asistencial de Adeslas, "nosotros gastamos menos y hacemos más", lo cual es una forma elegante de decir que el sector privado tiene mayor productividad que el sector público en la producción de servicios sanitarios homogéneos. Algunos directivos de las grandes empresas aseguradoras manifiestan, explícita o implícitamente, que el modelo MUFACE podría ser elegido por muchos ciudadanos; por ejemplo, D. Luis Monset, Director Gerente de ACES, en el año 2010 afirmó que "si los ciudadanos tuvieran capacidad legal de elegir, también elegirían, como los funcionarios, una póliza de aseguramiento privado. Esta situación no ocurre y es incomprensible, ya que el aseguramiento y provisión privados son más eficientes en términos económicos y de gestión, no generando déficit" ${ }^{29}$.

Si el modelo MUFACE tiene una larga trayectoria de éxito tanto en lo que respecta a la eficacia como a la eficiencia, ¿por qué los poderes públicos de las Comunidades Autónomas no han implantado un modelo similar? Asimismo, ¿por qué la mayoría de los expertos en Economía Pública ${ }^{30}$ o de la Salud, sabiendo que el modelo MUFACE es tan, o más, eficaz y eficiente que los SPRS no lo mencionan cuando realizan propuestas para hacer viable económica y financieramente el actual SNS?

${ }^{29}$ XVII Congreso Nacional de Derecho Sanitario. Publicado el 16-11-2010 en: http://www.fundacionidis.com/los-modelos-de-eleccion-como-muface-son-los-que-dan-mejores-respuestas-a-los-usuarios/

${ }^{30}$ Muchos de ellos son funcionarios públicos y, por tanto, como la gran mayoría, optan por las empresas privadas (Adeslas, Asisa, DKV y Sanitas en el 2012). 
En opinión del autor, se podría extender o implantar el modelo MUFACE en una primera fase, a todos los funcionarios de la Administración local y, en una segunda fase, a otros colectivos adscritos al Régimen General de la Seguridad Social (por ejemplo, al personal laboral de todas las Administraciones Públicas). Si se aplicará este modelo, aunque sólo fuera en lo referente a la asistencia sanitaria, el ahorro ${ }^{31}$ que se produciría para el erario público sería sustancial ya que la diferencia o exceso de coste del sector público es considerable (entre 300 y $500 €$ per cápita). Para que se pueda tener una idea aproximada de la dimensión de dicho ahorro en asistencia sanitaria, si se extendiera a toda la población protegida por los SPRS, dicho ahorro anual ascendería a 18.071 millones de euros ${ }^{32}$.

\section{CONCLUSIONES}

El modelo MUFACE consiste en que hay un millón y medio de españoles que tienen derecho a la "libre elección" entre la sanidad pública y la sanidad privada, siendo neutro para los mutualistas el coste de dicha elección. La gran mayoría elige recibir la prestación de los servicios sanitarios a través de las empresas privadas aseguradoras (EPSM). Estos mutualistas (activos, beneficiarios y pensionistas) tienen gratuitamente una "única" sanidad (los SPRS o EPSM), mientras que muchas personas a las que obligatoriamente se las adscribe a la sanidad pública (producción pública), por motivos diversos, tienen "doble cobertura", es decir, suscriben pólizas de seguros médicos con las empresas privadas aseguradoras y/o pagan directamente a los profesionales médicos por cada acto médico (visitas, intervenciones quirúrgicas, etc.).

Además, cada año, a lo largo del mes de enero, los mutualistas deciden si desean ser atendidos por los SPRS (sector público) o por una de las empresas aseguradoras privadas (Adeslas, Asisa, Igualatorio Cantabria y DKV, en 2015). La cobertura garantizada por las empresas aseguradoras privadas son equivalentes a las de la sanidad pública (la Cartera Común de Servicios del SNS) e incluso más prestaciones (por ejemplo, una limpieza de boca al año, aplicación de flúor tópico, obturaciones o empates para todos los niños menores de 15 años, habitaciones individuales y cama de acompañante en los hospitales, etc.).

${ }^{31}$ El modelo sanitario público español, tipo Beveridge, se financia con los impuestos que pagan todos los ciudadanos y, obviamente, unos ciudadanos contribuyen más que otros (según su capacidad de renta). Los que más contribuyen a la financiación son los que menos utilizan los servicios sanitarios públicos, bien porque tienen mejor salud (así lo corroboran múltiples estudios a nivel nacional e internacional) o bien porque paga voluntariamente un seguro privado que utilizan en la mayoría de las ocasiones, aunque tengan derecho a la asistencia sanitaria pública.

${ }^{32} \mathrm{Si}$ estimamos un ahorro medio per cápita de $400 €$ anuales y teniendo en consideración la población protegida en 2013 (45.178.685 personas), el ahorro ascendería a 18.071 millones de personas (400 x 45.178.685). 
Por otro lado, las EPSM no seleccionan el riesgo lo que permite afirmar que es incorrecto el mito de que las empresas aseguradoras privadas seleccionan a las personas jóvenes y sanas y la sanidad pública la eligen las mayores y enfermas. Además, se ha demostrado que el modelo MUFACE es más eficaz que el modelo imperante en las Comunidades Autónomas (los SPRS) y más eficiente que la sanidad pública ya que produce los mismos servicios sanitarios entre un $40 \%$ y 60\% más baratos, proporciona al usuario mayor libertad de elección de médico, especialista y centro hospitalario y, por último, mayor accesibilidad al servicio dado que las listas de espera son inferiores.

\section{BIBLIOGRAFÍA}

ABASOLO, I. y otros (2008), "Equidad en la utilización de servicios sanitarios públicos por Comunidades Autónomas en España: un análisis multinivel", Hacienda Pública Española, núm. 187 (4/2008), pp. 87-108.

BAREA, J. y GÓMEZ, A. (1999), El problema de la eficiencia del sector público en España. Especial consideración de la sanidad, Instituto de Estudios Económicos, Madrid.

FERNANDEZ PIRLA, J. M. (1974), "Economía y Gestión de la empresa", ediciones ICE, Madrid.

HOSPIDIO, L. y MORAL-BENITO, E. (2015), "Algunos determinantes de las diferencias entre los sectores público y privado en España”, Boletín Económico del Banco de España, febrero 2015, pp. 19-25.

ICEA (2012, 2013, 2014), Volumen y crecimiento de primas, ingresos derivados de la actividad, asegurados e importe de las prestaciones pagadas, de los años del 2012 al 2014. Disponible en: $h t t p: / / w w w . i c e a . e s / e s-E S / P a g i n a s / h o m e . a s p x$

INFORME PARA EL ANÁLISIS DEL GASTO SANITARIO (2005), Grupo de trabajo de la Conferencia de Presidentes para el análisis del gasto sanitario presidido por la IGAE. Disponible en: http://www.msssi.gob.es/ estadEstudios/estadisticas/sisInfSanSNS/infAnalisGasto.htm

LIMARQUEZ, M. (2004), "La asistencia sanitaria", en el IX Congreso Internacional del CLAD sobre Reforma del Estado y de la Administración Pública, celebrado en Madrid del 2 al 5 de noviembre de 2004. Disponible en http://siare.clad.org/fulltext/0049733.pdf.

LOPEZ SALUDAS, JM. (2013a), "La gestión privada de los servicios sanitarios públicos: los modelos Alzira y Madrid", Revista icade, núm. 90, septiembre-diciembre, pp. 33-53

LOPEZ SALUDAS, JM (2013b), "Análisis de la eficacia y la eficiencia del Servicio Nacional de Salud”, Boletín Económico ICE, núm. 3044, pp. 59-71. 
LOPEZ SALUDAS, JM (2013c), "Producción pública, gestión privada y eficiencia económica del Servicio Nacional de Salud", Tesis doctoral dirigida por el Dr. Leopoldo Gonzalo y González presentada en el Departamento de Economía Aplicada de la UNED. Disponible en: http://e-spacio.uned. es/fez/view/tesisuned: CiencEcoEmp-Jmlopez.

MINISTERIO DE SANIDAD, SERVICIOS SOCIALES E IGUALDAD (2015a), Barómetro Sanitario 2013. Disponible en:

http://www.msssi.gob.es/estadEstudios/estadisticas/docs/BS_2013/ BS_2013Presentacion.pdf

MINISTERIO DE SANIDAD, SERVICIOS SOCIALES E IGUALDAD (2015b), Estadística del Gasto Sanitario Público (EGSP) 2002-2013. Disponible en:

http://www.msssi.gob.es/estadEstudios/estadisticas/inforRecopilaciones/gastoSanitario2005/home.htm

MINISTERIO DE SANIDAD, SERVICIOS SOCIALES E IGUALDAD (2015c), Sistema de Cuentas de Salud 2012. Disponible en:

http://www.msssi.gob.es/estadEstudios/estadisticas/sisInfSanSNS/pdf/SCSdatosEstadisticos.pdf

MUFACE, Memoria Anual de los años 2007 al 2013.

MUSGRAVE, R. A. (1969), Teoría de la Hacienda Pública, Editorial Aguilar, Madrid.

PRICEWATERHOUSECOOPERS (2010), "Quince temas candentes de la Sanidad española para 2010". Disponible en:

https://mesaendefensasanidadpublica.files.wordpress.com/2013/05/pwc_informe-15-temas-candentes-sanidad-espac3b1ola_2010.pdf

RAZQUIN, P. M. (1998), "El seguro de salud. Escenario actual y perspectivas", Boletín del Círculo de Empresarios, núm. 63, pp. 259-283.

ROSEN, H. S. (2002), Hacienda Pública. $5^{a}$ edición, McGraw Hill, Madrid. 
